from the ohmic behavior that is observed in pure InAs whiskers. Additional details on the electronic properties of these structures are reported in the February 11 issue of Applied Physics Letters.

To synthesize $\mathrm{Si} / \mathrm{SiGe}$ nanowires with diameters ranging from $50 \mathrm{~nm}$ to $300 \mathrm{~nm}$, $\mathrm{Wu}$ and co-workers periodically introduced Ge vapor through pulsed laser ablation of a Ge target in the presence of a $\mathrm{Au}$ nanocluster catalyst. At temperatures ranging from $850^{\circ} \mathrm{C}$ to $950^{\circ} \mathrm{C}$, a $\mathrm{Au}$ thin film on a Si substrate forms an alloy with Si and separates into nanometersized droplets. Si continuously deposits into the Au-Si alloy droplets, and growth of the Si nanowire occurs upon supersaturation of the droplets. By turning the laser on, the researchers caused both Ge and $\mathrm{Si}$ to be deposited into the droplets, causing the precipitation of SiGe. By continuously switching the laser on and off, a $\mathrm{Si} / \mathrm{SiGe}$ superlattice was formed in a block-by-block fashion. The researchers showed that the diameter and composition of the highly crystalline $\mathrm{Si} / \mathrm{SiGe}$ nanowires could be controlled by adjusting the reaction conditions. Specifically, the nanowire diameter was varied from $20 \mathrm{~nm}$ to $100 \mathrm{~nm}$ by changing the thickness of the Au film from $1 \mathrm{~nm}$ to $20 \mathrm{~nm}$.

The work performed by these three research teams signifies an important turning point in nanoscale research. Analogous to the way in which twodimensional thin-film heterostructures transformed the planar semiconductor industry, heterostructures created inside nanowires offer the potential for diverse applications such as nanobarcodes, polarized nanoscale LEDs, 1D-0D-1D resonant tunneling devices, and improved thermoelectric devices.

STEFFEN K. KALDOR

\section{Simple Method Can Suspend Individual Nanofibers}

Researchers at Germany's Max Planck Institute have devised a technique to suspend an individual nanofiber over a $\mathrm{Si} / \mathrm{SiO}_{2}$ substrate by using coordinate markers and a sacrificial layer of electronbeam resist. Individual suspended nanofibers are required to study their electromechanical properties. The generality of this method is of key importance because it does not rely on the selectivity of particular etching processes or on the necessity of growing fibers by means of chemical vapor deposition. Gyu-Tae Kim and colleagues report in the March 11 issue of Applied Physics Letters that they have demonstrated their technique by suspending a 2.3-nm-diameter carbon nanotube and measuring its Young's modulus by using a calibrated atomic force microscope (AFM) tip.

To achieve this suspended-fiber configuration, Kim and co-workers prepared a $\mathrm{Si} / \mathrm{SiO}_{2}$ substrate with reference marks made by means of electron-beam deposition. They spin-coated this substrate with an electron-beam resist, poly(methyl methacrylate) (PMMA), then spindeposited a solution that contained dispersed nanofibers. The researchers determined the locations of these fibers relative to the underlying coordinate system using AFM. More PMMA was spun on, effectively embedding and fixing the strands parallel to the substrate, much as amber might fix a fly. Next, a directed electron beam exposed the layers of resist at the head and tail of a selected nanofiber, and a PMMA developer removed the treated resist, forming two holes in the PMMA that opened through to the $\mathrm{Si} / \mathrm{SiO}_{2}$ underneath. At this point, the ends of the fiber protruded from the side of each of the pits so formed. The researchers then deposited a $\mathrm{Au} / \mathrm{Pd}$ film that surrounded and cemented the now-encased fiber ends to the substrate beneath. They took care to deposit from two directions in order to prevent the nanofiber ends from shadowing the underlying $\mathrm{Si} / \mathrm{SiO}_{2}$. Finally, a liftoff procedure removed all of the remaining PMMA from around and under the fiber, leaving the now-suspended nanofiber spanning two metal blocks. When the distance between the metal posts exceeded $500 \mathrm{~nm}$, most of the suspended nanotubes sagged to the point of touching the substrate.

Research into the electromechanical properties of nanofibers requires the suspended configuration, and this may also be an effective system for other studies, such as the synthesis of molecular electronic devices. The scientists said that their method can be applied to many different fibers, and will allow the comparison of a suspended fiber to one lying on the substrate.

RiCHARD N. LOUIE

\section{Internal Defects Observed by Two-Photon-Induced Photoluminescence}

Structural imperfections in semiconductors play an important role in areas of high-efficiency emissive materials such as laser diodes. Researchers in the Department of Engineering at Shizuoka University in Japan have generated threedimensional (3D) images of such imperfections through the first reported use of two-photon excitation, achieved using a laser scanning microscope. Y. Kawata and co-workers imaged regions of bulk polycrystalline ZnSe up to $200 \mu \mathrm{m}$ below the surface by measuring the effect of position on photoluminescence (PL).

According to Kawata, "[T]wo-photon excitation is a significant improvement over conventional PL observation techniques, which allow only the surface defects to be observed because of the large absorption of the excitation light."

As reported in the March 1 issue of Optics Letters, the researchers used a 200-mW Ti:sapphire laser generating $80-\mathrm{fs}$ pulses at $790 \mathrm{~nm}$. The laser light was focused to a small spot by using a beam expander and an objective lens. The $5 \mathrm{~mm} \times 5 \mathrm{~mm} \times 3 \mathrm{~mm}$ polycrystalline $\mathrm{ZnSe}$ sample was mounted on a stage capable of $3 \mathrm{D}$ translation with 50-nm precision. The stage was used to position the polycrystal relative to the focused laser spot. Because the energy of the laser light is far below the bandgap energy of the semiconductor, the light is absorbed only in the focused region where the probability of simultaneous absorption of two photons is high. A dichroic mirror and interference filter were used to remove the exciting radiation from the PL before the intensity was measured with a photomultiplier tube. A defect map of the polycrystal was created by moving the stage and recording the PL intensity as a function of the position of the focused spot. The impurities, cavities, cracks, and other defects within the polycrystal absorbed or diffracted the PL and were seen as dark regions in the scanned images.

The depth limit of the two-photon technique results primarily from the refraction-induced spherical aberration of the exciting radiation inside the polycrystal. This aberration increases the laser spot size at focus, thus lowering the PL intensity and the contrast in the scanned images. Using higher laser power in an effort to increase the PL intensity results in photo damage to the semiconductor sample. The researchers are currently investigating the use of a liquid-crystal phase mask to reduce the spherical aberration.

GREG KHITROV

\section{Lateral Color Integration Achieved with Rare-Earth-Doped GaN}

D.S. Lee and A.J. Steckl of the Nanoelectronics Laboratory at the University of Cincinnati have used a rare-earthdoped GaN host to produce laterally integrated electroluminescent devices (ELDs) with red and green emissions. While vertical integration (deposition of different dopants on different layers) has provided a satisfactory visible spectrum, each dopant layer demands a different biasing condition in order to produce its representative color. Such layer-by-layer 